\title{
Microglial activation and neuroinflammation in Alzheimer's disease: a critical examination of recent history
}

\section{Wolfgang J. Streit*}

Department of Neuroscience, University of Florida College of Medicine, Gainesville, FL, USA

\section{Edited by:}

Elena Galea, Universitat Autònoma de

Barcelona, Spain

\section{Reviewed by:}

Carme Sola, Instituto de Investigaciones

Biomédicas de Barcelona-CSIC, Spain

Elena Galea, Universitat Autònoma de

Barcelona, Spain

\section{*Correspondence:}

Wolfgang J. Streit, Department of

Neuroscience, P.O. Box 100244

University of Florida College of

Medicine, Gainesville, FL 32610-0244,

USA.

e-mail:streit@mbi.ufl.edu
The neurofibrillary degeneration that occurs in Alzheimer's disease (AD) is thought to be the result of a chronic and damaging neuroinflammatory response mediated by neurotoxic substances produced by activated microglial cells. This neuroinflammation hypothesis of AD pathogenesis has led to numerous clinical trials with anti-inflammatory drugs, none of which have shown clear benefits for slowing or preventing disease onset and progression. In this paper, I make the point that $A D$ is not an inflammatory condition, and reconstruct the sequence of events during the 1980s and 1990s that I believe led to the development of this faulty theory.

Keywords: major histocompatibility complex antigens, cytokines, amyloid, aging, chronic inflammation

\section{INTRODUCTION}

One might reasonably argue that a microglial renaissance occurred during the mid-to late 1980s. Although anatomists of the early 20th century, such as Nissl and del Rio Hortega, had already made seminal contributions toward understanding microglial biology, for reasons unknown microglia remained largely hidden away from mainstream neuroscience for much of the decades following these early studies. During the 1970s and well into the 1980s the one key issue dominating microglial neurobiology concerned their embryological origin, i.e., whether the cells are of mesodermal or neuroectodermal origin (Theele and Streit, 1993). Many would now consider this question resolved in favor of a mesodermal lineage, but rapid developments in the field of neural precursor cells may once again bring the ontogeny issue to the forefront for reconsideration.

The rebirth of microglia in the 1980s occurred because of two major developments: methods for culturing microglial cells in vitro and reagents for visualizing cells in situ became available. These advances were critical for stimulating renewed interest in the almost forgotten "third element" of Cajal as is abundantly evident now, some 20 years later, by the explosion of published papers on microglial cells. However, excitement over the rediscovery of microglia also produced a major misconception regarding functional roles of activated microglia, namely, that microglial activation is harmful to neurons in the CNS. Thus, with regard to the question posed in this special issue: where did we get lost, the short answer is, we got lost early on by misinterpreting biological functions of microglia. The purpose of this paper is to describe how this misconception came about and how it became entangled with the concept of detrimental neuroinflammation which is now believed by many to underlie not only Alzheimer's disease (AD) pathogenesis but a number of other major neurodegenerative conditions, including Parkinson's disease, amyotrophic lateral sclerosis and HIV-associated dementia.

\section{CONCEPTUAL ERRORS IN THE INTERPRETATION OF MICROGLIAL ACTIVATION}

The initial understanding of what constitutes an activated microglial cell was simple and straightforward - activated microglia were identified as reactive mononuclear elements responding to acute CNS injury in vivo (Oehmichen and Huber, 1976). These experiments represented a modern day extension of classic experiments performed by Metchnikoff in the late 1800s in that they were focused on studying the inflammatory response, only this time in the brain. There was little doubt that activated microglia were part of an acute cellular response to an injurious stimulus, which for Oehmichen and Huber consisted of implanting glass cover slips into the rabbit brain. A more sophisticated paradigm of eliciting microglial activation whose origins date back to the work of Nissl at the turn of the 20th century, and advocated by Kreutzberg and colleagues beginning in the 1960s was the facial nerve axotomy paradigm. In this model, an acute and local neuroinflammatory response (microglial activation) can be induced in the facial nucleus simply by cutting the peripheral axons of cranial nerve VII. Although Kreutzberg and colleagues did not use the term "inflammation" but usually spoke of microglial activation, it was implicit because microglial activation represents a cellular reaction to injury, which in essence constitutes the definition of inflammation according to textbooks of pathology. Importantly, axotomy-induced microglial activation is associated both temporally and spatially with the successful regeneration of axotomized motoneurons, providing an unambiguous association between microglial activation and the natural wound healing response, and clearly supporting a beneficial role for activated microglia.

The concept of microglial activation changed in the 1980s when researchers began to model it in vitro. Pioneering work done by Giulian and Baker (1986) established a relatively simple procedure for isolating and maintaining microglial cells in the culture dish, and their method quickly became the procedure of choice 
for many other laboratories interested in studying the biological functions of microglia. In their initial as well as in subsequent papers, Giulian and colleagues emphasized the production of a prototypical proinflammatory cytokine, interleukin-1 (IL-1), by ameboid microglia (so-called because of their resemblance to macrophages), which was reaffirmed by Hetier et al. (1988) just a couple of years later by showing that IL-1 mRNA synthesis was induced in these cultured cells following their stimulation with bacterial lipopolysaccharide (LPS). With this introduction to neurobiology of LPS-mediated macrophage activation, already well-established and accepted in immunology circles, the flood gates were opened for studying microglial activation in vitro. Countless studies have since then been performed using various modifications of the LPS model as well as other immunostimulatory paradigms for studying activated microglia in vitro. However, most of these studies have not taken into consideration the fact that microglia activated by LPS in vitro behave very differently than microglia activated by injured neurons in the brain. We have previously discussed this discrepancy between in vivo and in vitro concepts of microglial activation (Streit et al., 1999), pointing out that the generation of microglial cell cultures involves extreme brain damage (chopping and trituration of dissected tissues) inevitably causing immediate activation of microglia and their transformation into brain macrophages. When these activated microglia-derived brain macrophages are additionally stimulated with LPS, the result is superactivated cells that produce not only copious quantities of IL-1 but many other secretory products. It seems that cells treated in this manner are activated to a maximal extent and are near the breaking point; indeed more recent work shows that LPS-mediated overactivation leads to microglial cell death (Liu et al., 2001). Thus, one key juncture where we got lost was in the silent assumption that cultured microglia unstimulated by LPS were representative of resting microglia in the normal brain, and that LPS-activated cells were analogous to activated microglia in the damaged brain. Any extrapolations made from such in vitro data are therefore unlikely to apply to most in vivo scenarios of microglial activation with the possible exception of a bacterial CNS infection. Case in point, ameboid microglia in vivo do not produce IL-1 (Hurley et al., 1999), as suggested by in vitro studies (Giulian et al., 1986). Moreover, the in vitro findings on microglial IL-1 production likely caused many to assume that whenever activated microglia were present in situ the cells produced IL- 1 in a sustained manner. However, IL-1 production in vivo varies substantially and even in cases of severe CNS damage, such as spinal cord trauma, the duration of IL-1 production is limited to the first $24 \mathrm{~h}$ post-injury after which it drops to control levels (Streit et al., 1998). During the acute neuroinflammatory reaction in response to facial nerve axotomy IL-1 mRNA levels are very low and essentially the same as in uninjured control tissues (Streit et al., 1998). In addition to IL-1, which is often thought of as a potentially harmful cytokine associated with chronic inflammatory states, such as rheumatoid arthritis, other potentially damaging substances were found to be produced by activated microglia in vitro. Perhaps most notable and influential in this regard was the early study by Colton and Gilbert (1987) showing that microglia activated by zymosan can produce superoxide anions. Although the authors at the time presented their findings in the context of a bactericidal action of activated microglia, the data were extrapolated later on by many others to support the idea that activated microglia contribute to oxidative stress in the injured and diseased brain. While in vitro studies continued to gain momentum and eventually produced a picture of microglia as neurotoxic effector cells (Boje and Arora, 1992; Chao et al., 1992; Giulian et al., 1993), other researchers were studying microglial activation in a variety of in vivo brain injury paradigms, as well as in the diseased human brain, taking advantage of the availability of new antibodies that allowed the visualization of microglia in situ.

Soon after the introduction of in vitro methods, McGeer et al. (1987) reported in a landmark paper the presence of reactive (activated) microglia in the brains of humans with AD. Their study together with another one by Rogers et al. (1988) 1 year later, and the in vitro data showing production of potentially harmful substances by activated microglia would set the stage for development of the notion that detrimental neuroinflammation plays a major role in AD pathogenesis. McGeer's discovery was made possible by the availability of antibodies directed against major histocompatibility complex (MHC) antigens, which are well-known recognition molecules essential for mediating specific cell-cell interaction in the immune system, most significantly antigen presentation. Their findings were spectacular because of a prevailing view at the time that the brain is immunologically quiescent, i.e., an immunologically privileged organ where lymphatic drainage and MHC antigen expression are absent. However, McGeer's identification of activated microglia by virtue of the fact that these cells were expressing MHC class II molecules was not entirely correct because MHC II expression occurs prominently also on non-activated microglia and perivascular cells in the normal human and animal brain (Craggs and Webster, 1985; Hayes et al., 1987; Streit et al., 1989). Nonetheless, the notion that MHC II expression can serve as an objective biomolecular marker for activated microglia was seemingly corroborated by subsequent animal studies that showed increases in MHC II expression on a subset of microglial cells following experimental lesions (Akiyama et al., 1988; Streit et al., 1989; Smetanka et al., 1990), encouraging its widespread acceptance. The idea that neuroinflammatory processes are involved in $\mathrm{AD}$ was buttressed further by additional findings from the McGeer group showing presence of complement proteins and integrins in the AD brain (McGeer et al., 1989; Akiyama and McGeer, 1990), as well as by findings showing presence of IL-1 immunoreactive microglia in Down's and AD cases (Griffin et al., 1989). Pretty soon, a large number of other inflammatory mediators, including many cytokines, were being added to the list of substances thought to be consistently increased in the $\mathrm{AD}$ brain and by the time a major review on $\mathrm{AD}$ and neuroinflammation was published in 2000 (Akiyama et al., 2000), the notion was firmly embedded in mainstream thinking. A potential problem with all of these studies focused on measuring various inflammatory molecules in the AD brain is that most if not all of the inflammatory proteins (similar to MHC antigens) are also expressed in non-AD brains, and that levels of these are likely to vary substantially in both populations depending on whether or not peripheral infections are present. Thus, any future work along these lines should exclude subjects with infectious comorbidities. 


\section{ACUTE VERSUS CHRONIC INFLAMMATION AND THE ROLE OF AMYLOID- $\beta$ PROTEIN}

Another place where we got lost was by not differentiating between acute and chronic inflammation and distinguishing between a wound healing response and autoimmune disease, although I will maintain that neither contributes to $\mathrm{AD}$ neurodegeneration. While acute inflammation comprises the immediate and early reaction to an injurious event and is basically an adaptive response that paves the way for repair of the damaged site, chronic inflammation results from injurious stimuli that are persistent. There is no evidence of any injurious event in $\mathrm{AD}$ that would precipitate an inflammatory reaction, although some might argue that amyloid- $\beta$ protein $(A \beta)$ qualifies in this regard (see below). In the periphery, both acute and chronic inflammation are characterized by leukocytic exudates consisting primarily of polymorphonuclear cells (neutrophils) in the former, and mononuclear cells (macrophages, lymphocytes, plasma cells) in the latter. In the CNS, because of the presence of the blood brain barrier, leukocytic exudates may or may not be part of acute neuroinflammation, the facial nerve axotomy paradigm being a premier example of this (Raivich et al., 1998). The classic example of chronic neuroinflammation occurring in the CNS is found in multiple sclerosis, a disease marked by prominent leukocytic infiltrates. While the underlying cause(s) of MS have not been identified, it is probably safe to say that the persistent injurious stimulus that accounts for MS neuroinflammation is a myelin-related protein that has escaped self-tolerance and become an autoantigen, which is consistent with how experimental autoimmune encephalomyelitis is induced in animals. With the chronic persistence of a CNS autoantigen there is a persistent accumulation of blood-derived mononuclear leukocytes in the CNS parenchyma, which mirrors the presence of leukocytic exudates in peripheral inflammatory diseases, such as rheumatoid arthritis or polymyositis. It is the persistent presence of extensive leukocytic infiltrates that eventually produces tissue damage. In $\mathrm{AD}$ there are no lymphocytic infiltrates to speak of, making it very difficult to maintain that $\mathrm{AD}$ is an inflammatory disease. Nonetheless, it was assumed that the ostensible inflammation in $\mathrm{AD}$ is of a chronic and therefore detrimental nature because $\mathrm{AD}$ is after all an aging-related condition and a number of studies had shown a progressive increase in the expression of MHC class II antigens in the normal aging brain (Rogers et al., 1988; Perry et al., 1993; Ogura et al., 1994; Sheffield and Berman, 1998), suggesting that normal aging is accompanied by inflammation. Also, the word "disease" implicitly carries the notion of chronicity and so it was assumed that whatever inflammatory changes were detected in $\mathrm{AD}$ had been there for some time when, in fact, it is impossible to say so from a one-time post-mortem histopathological observation. The idea that microglia are cytotoxic inflammatory cells that contribute towards neurodegeneration in $\mathrm{AD}$ was propagated and ostensibly corroborated through additional in vitro studies showing that when cultured microglial cells are stimulated with $A \beta$ peptides they produce molecules which are toxic to cultured neurons (Meda et al., 1995; Giulian et al., 1996; Combs et al., 1999). The conclusion that $\mathrm{A} \beta$ deposits are inflammatogenic in the $\mathrm{AD}$ brain and incite detrimental inflammatory activity was at this point all but inevitable, especially since it seemed to be consistent with numerous prior histopathological descriptions of microglial aggregations at amyloid plaques. $A \beta$ peptides had also been shown to be neurotoxic in vitro (Yankner et al., 1989), which probably served to encourage this line of reasoning, although in the same year and in the same journal a report appeared describing a neurotrophic effects of A $\beta$ peptides in vitro (Whitson et al., 1989). Conflicting findings like these underscore the potential fallacies associated with in vitro experimentation, where outcomes can be influenced profoundly by small variations in any one of a number of experimental parameters. In any event, a key question within the current context is, does the presence of amyloid deposits in $\mathrm{AD}$ brain represent a persistent injurious stimulus that incites chronic neuroinflammation? The answer is, probably not, because despite numerous histopathological studies claiming presence of microglial activation around amyloid deposits, a closer look at these allegedly activated cells has revealed that microglia associated with senile (neuritic) plaques more times than not display a dystrophic rather than an activated morphology (Streit et al., 2009). Moreover, the fact that microglia become clustered at senile plaques is by itself not indicative of an inflammatory reaction but instead is more likely to reflect an abnormal cellular reaction triggered by presence of amyloid fibrils. It is known that diffuse, non-fibrillar (early) amyloid deposits, even when extensive, do not elicit microglial changes (Ohgami et al., 1991; Mackenzie et al., 1995; Streit et al., 2009), and thus any microglial changes that have been associated with more advanced amyloid plaques are likely to be the result of altered chemistry of amyloid peptides. In addition, there is in vivo evidence showing increased presence of $A \beta$ and amyloid precursor protein after traumatic head injury (Smith et al., 2003) strongly supporting the view that increased $\mathrm{A} \beta$ production in $\mathrm{AD}$ is the result rather than the cause of CNS disturbances. At the same time, it would be a stretch to postulate that extracellular deposition of amyloid peptides in and of itself constitutes an injury.

So, how does one reconcile the many histopathological descriptions of activated microglia in $\mathrm{AD}$ with the claim made here that $\mathrm{AD}$ is not an inflammatory disease? First, if indeed microglial activation does occur at some point during the evolution of senile plaques, it does not necessarily have to be detrimental and cause AD neurofibrillary degeneration. Neurofibrillary tangles and neuropil threads are not limited to the vicinity of amyloid deposits and they occur during normal aging, as well as in other neurological diseases and in the absence of amyloid. Second, there is a good chance that nonactivated (especially dystrophic) microglia were misidentified as activated cells based not only on the false assumption that MHC II expression is an immunological marker for activated cells, but also because of only a superficial assessment of their morphology. In the late 1980s and early 1990s the idea of morphologically abnormal (dystrophic) microglia did not exist and with the excitement over the rediscovery of microglia researchers were quick to identify any non-ramified microglial cell as activated. Lastly, the possibility that microglial activation observable within the $\mathrm{AD}$ brain might be the result of infectious disease in the periphery has never been studied systematically; in other words, studies that have assessed neuroinflammation in $\mathrm{AD}$ did not make a distinction between $\mathrm{AD}$ cases with and without infectious disease comorbidities, which are quite common in $\mathrm{AD}$ patients. We know now that peripheral infections in humans are accompanied by prominent microglial activation 
(Lemstra et al., 2007; Streit et al., 2009), and it is certainly conceivable that other, less severe complications, such as pneumonia, could influence microglial activation.

\section{CONCLUSIONS}

To sum up briefly where we got lost, most significant was the overinterpretation of cell culture data and the indiscriminate extrapolation of microglial cytotoxin production from an artificial in vitro environment to the human brain. There was a clear failure to recognize the disparity between microglial activation, as produced in vitro through LPS or other immune stimulation,

\section{REFERENCES}

Akiyama, H., Barger, S., Barnum, S., Bradt, B., Bauer, J., Cole, G. M., Cooper, N. R., Eikelenboom, P., Emmerling, M., Fiebich, B. L., Finch, C. E., Frautschy, S., Griffin, W. S., Hampel, H., Hull, M., Landreth, G., Lue, L., Mrak, R., Mackenzie, I. R., McGeer, P. L., O’Banion, M. K., Pachter, J., Pasinetti, G., Plata-Salaman, C., Rogers, J., Rydel, R., Shen, Y., Streit, W., Strohmeyer, R., Tooyoma, I., Van Muiswinkel, F. L., Veerhuis, R., Walker, D., Webster, S., Wegrzyniak, B., Wenk, G., and WyssCoray, T. (2000). Inflammation and Alzheimer's disease. Neurobiol. Aging 21, 383-421.

Akiyama, H., Itagaki, S., and McGeer, P. L. (1988). Major histocompatibility complex antigen expression on rat microglia following epidural kainic acid lesions. J. Neurosci. Res. 20, 147-157.

Akiyama, H., and McGeer, P. L. (1990). Brain microglia constitutively express beta-2 integrins. J. Neuroimmunol.30, 81-93.

Boje, K. M., and Arora, P. K. (1992). Microglial-produced nitric oxide and reactive nitrogen oxides mediate neuronal cell death. Brain Res. 587, 250-256.

Chao, C. C., Hu, S., Molitor, T. W., Shaskan, E. G., and Peterson, P. K. (1992). Activated microglia mediate neuronal cell injury via a nitric oxide mechanism. J. Immunol. 149, 2736-2741.

Colton, C. A., and Gilbert, D. L. (1987). Production of superoxide anions by a CNS macrophage, the microglia. FEBS Lett. 223, 284-288.

Combs, C. K., Johnson, D. E., Cannady, S. B., Lehman, T. M., and Landreth, G. E. (1999). Identification of microglial signal transduction pathways mediating a neurotoxic response to amyloidogenic fragments of beta-amyloid and prion proteins. J. Neurosci. 19, 928-939.

Craggs, R. I., and Webster, H. D. (1985). Ia antigens in the normal rat nervous system and in lesions of experimental allergic encephalomyelitis. Acta Neuropathol. 68, 263-272.

Giulian, D., and Baker, T. J. (1986). Characterization of ameboid microglia isolated from developing mammalian brain. J. Neurosci. 6, 2163-2178.

Giulian, D., Baker, T. J., Shih, L. C., and Lachman, L. B. (1986). Interleukin 1 of the central nervous system is produced by ameboid microglia. J. Exp. Med. 164, 594-604.

Giulian, D., Haverkamp, L. J., Yu, J. H., Karshin, W., Tom, D., Li, J., Kirkpatrick, J., Kuo, L. M., and Roher, A. E. (1996). Specific domains of beta-amyloid from Alzheimer plaque elicit neuron killing in human microglia. J. Neurosci. 16, 6021-6037.

Giulian, D., Vaca, K., and Corpuz, M. (1993). Brain glia release factors with opposing actions upon neuronal survival. J. Neurosci. 13, 29-37.

Griffin, W. S., Stanley, L. C., Ling, C., White, L., MacLeod, V., Perrot, L. J., White, C. L. III, and, Araoz, C. (1989). Brain interleukin 1 and S-100 immunoreactivity are elevated in Down syndrome and Alzheimer disease. Proc. Natl. Acad. Sci. U.S.A. 86, 7611-7615.

Hayes, G. M., Woodroofe, M. N., and Cuzner,M.L. (1987). Microglia are the major cell type expressing MHC class II in human white matter. J. Neurol. Sci. 80, 25-37.

Hetier, E., Ayala, J., Denefle, P., Bousseau, A., Rouget, P., Mallat, M., and Prochiantz, A. (1988). Brain macrophages synthesize interleukin-1 and interleukin-1 mRNAs in vitro. $J$. Neurosci. Res. 21, 391-397.

Hurley, S. D., Walter, S. A., SempleRowland, S. L., and Streit, W. J. (1999). Cytokine transcripts expressed by microglia in vitro are not expressed by ameboid microglia of the developing rat central nervous system. Glia 25, 304-309.

Lemstra, A. W., Groen in't Woud, J. C. M., Hoozemans, J. J. M., van Haastert, E. S., Rozemuller, A. J. M., Eikelenboon, P., and van Gool, W.A. (2007). Microglia

and microglial activation as it occurs in vivo. Related to this was the swift and categorical acceptance of MHC expression as an objective marker for activated microglia in vivo. Finally, the assumption was made that any inflammation-like changes reported in $\mathrm{AD}$ were indicative of a chronic and therefore detrimental process that was causative in the development of neurodegeneration.

\section{ACKNOWLEDGMENT}

Supported by a grant from the National Institutes of Health, AG023665.

activation in sepsis: a case-control study. J. Neuroinflammation 4, 4.

Liu, B., Wang, K., Gao, H. M., Mandavilli, B., Wang, J. Y., and Hong, J. S. (2001). Molecular consequences of activated microglia in the brain: overactivation induces apoptosis. J. Neurochem. 77, 182-189.

Mackenzie, I. R., Hao, C., and Munoz, D. G. (1995). Role of microglia in senile plaque formation. Neurobiol. Aging 16, 797-804.

McGeer, P. L., Akiyama, H., Itagaki, S., and McGeer,E.G. (1989). Activation of the classical complement pathway in brain tissue of Alzheimer patients. Neurosci. Lett. 107, 341-346.

McGeer, P. L., Itagaki, S., Tago, H., and McGeer, E. G. (1987). Reactive microglia in patients with senile dementia of the Alzheimer type are positive for the histocompatibility glycoprotein HLA-DR. Neurosci. Lett. 79 , 195-200.

Meda, L., Cassatella, M. A., Szendrei, G. I., Otvos, L. Jr., Baron, P., Villalba, M., Ferrari, D., and Rossi, F. (1995). Activation of microglial cells by beta-amyloid protein and interferongamma. Nature 374, 647-650.

Oehmichen, M., and Huber, H. (1976). Reactive microglia with membrane features of mononuclear phagocytes. J. Neuropathol. Exp. Neurol. 35, 30-39.

Ogura, K., Ogawa, M., and Yoshida, M. (1994). Effects of ageing on microglia in the normal rat brain: immunohistochemical observations. Neuroreport 5, 1224-1226.

Ohgami, T., Kitamoto, T., Shin, R. W., Kaneko, Y., Ogomori, K., and Tateishi, J. (1991). Increased senile plaques without microglia in Alzheimer's disease. Acta Neuropathol. 81, 242-247.

Perry, V. H., Matyszak, M. K., and Fearn, S. (1993). Altered antigen expression of microglia in the aged rodent CNS. Glia 7, 60-67.

Raivich, G., Jones, L. L., Kloss, C. U., Werner, A., Neumann, H., and Kreutzberg, G. W. (1998). Immune surveillance in the injured nervous system: T-lymphocytes invade the axotomized mouse facial motor nucleus and aggregate around sites of neuronal degeneration. J. Neurosci. 18 , 5804-5816.

Rogers, J., Luber-Narod, J., Styren, S. D., and Civin, W.H. (1988). Expression of immune system-associated antigens by cells of the human central nervous system: relationship to the pathology of Alzheimer's disease. Neurobiol. Aging 9, 339-349.

Sheffield, L. G., and Berman, N. E. (1998). Microglial expression of MHC class II increases in normal aging of nonhuman primates. Neurobiol. Aging 19, 47-55.

Smetanka, A. M., Yee, K. T., and Lund, R. D. (1990). Differential induction of class I and II MHC antigen expression by degenerating myelinated and unmyelinated axons. Brain Res. 521, 343-346.

Smith, D. H., Uryu, K., Saatman, K. E., Trojanowski, J. Q., and McIntosh, T.K. (2003). Protein accumulation in traumatic brain injury. Neuromolecular Med. 4, 59-72.

Streit, W. J., Braak, H., Xue, Q. S., and Bechmann, I. (2009). Dystrophic (senescent) rather than activated microglial cells are associated with tau pathology and likely precede neurodegeneration in Alzheimer's disease. Acta Neuropathol. 118, 475-485.

Streit,W.J., Graeber,M.B., and Kreutzberg, G. W. (1989). Expression of Ia antigen on perivascular and microglial cells after sublethal and lethal motor neuron injury. Exp. Neurol. 105, 115-126.

Streit, W. J., Semple-Rowland, S. L., Hurley, S. D., Miller, R. C., Popovich, P.G., and Stokes, B. T. (1998). Cytokine mRNA profiles in contused spinal cord and axotomized facial nucleus suggest a beneficial role for inflammation and gliosis. Exp. Neurol. 152, 74-87.

Streit, W. J., Walter, S. A., and Pennell, N. A. (1999). Reactive microgliosis. Prog. Neurobiol. 57, 563-581.

Theele, D. P., and Streit, W. J. (1993). A chronicle of microglial ontogeny. Glia 7, 5-8. 
Whitson,J.S.,Selkoe,D.J., and Cotman,C.W. (1989). Amyloid beta protein enhances the survival of hippocampal neurons in vitro. Science 243, 1488-1490.

Yankner, B.A., Dawes, L. R., Fisher,S., VillaKomaroff, L., Oster-Granite, M.L., and Neve, R. L. (1989). Neurotoxicity of a fragment of the amyloid precursor associated with Alzheimer's disease. Science 245, 417-420.

Conflict of Interest Statement:The author declares that the research was conducted in the absence of any commercial or financial relationships that could be construed as a potential conflict of interest.
Received: 03 March 2010; paper pending published: 06 April 2010; accepted: 04 May 2010; published online: 03 June 2010.

Citation: Streit WJ (2010) Microglial activation and neuroinflammation in Alzheimer's disease: a critical examination of recent history. Front. Ag. Neurosci. 2:22. doi: 10.3389/fnagi.2010.00022
Copyright () 2010 Streit. This is an open-access article subject to an exclusive license agreement between the authors and the Frontiers Research Foundation, which permits unrestricted use, distribution, and reproduction in any medium, provided the original authors and source are credited. 